\title{
Effect of nicorandil administration on myocardial microcirculation during primary percutaneous coronary intervention in patients with acute myocardial infarction
}

\author{
Chunguang Feng, Bing Han, Yi Liu, Lulu Wang, Dongdong Niu, Ming Lou, Cunzhi Lu \\ Institute of Cardiovascular Disease, Xuzhou Central Hospital, Xuzhou, China
}

Adv Interv Cardiol 2018; 14, 1 (51): 26-31

DOI: https://doi.org/10.5114/aic.2018.74352

\begin{abstract}
A bstract
Introduction: Prevention of the no-reflow phenomenon has a crucial role in primary percutaneous coronary intervention (P-PCI) procedures.

Aim: To assess the effects of early intracoronary administration of nicorandil (NIC) during P-PCI on myocardial microcirculation in patients with acute myocardial infarction (AMI).

Material and methods: A total of 120 patients with first acute anterior wall ST segment elevation myocardial infarction who underwent P-PCI were randomly divided into two groups: the NIC group $(\mathrm{A}, n=60)$ and the placebo group $(\mathrm{B}, n=60)$. Before stent placement, NIC or normal saline was injected using a guiding catheter. The thrombolysis in myocardial infarction (TIMI) grade, TIMI myocardial perfusion grade (TMPG), resolution of ST segment elevation (defined as $>50 \%$ decrease in ST elevation) $1 \mathrm{~h}$ after surgery, and ${ }^{99} \mathrm{TC}^{\mathrm{m}}$-methoxyisobutyl isocyanide (MIBI) rest myocardial perfusion imaging (MPI) via single-photon emission computed tomography $\left({ }^{99} \mathrm{TC} \mathrm{C}^{\mathrm{m}}\right.$-MIBI SPECT) findings 10 days after surgery were compared between the two groups.

Results: The number of patients who achieved TIMI grade 3 (96.67\% vs. 86.67\%; $p=0.047)$ and TMPG 3 (95\% vs. $83.33 \% ; p=$ 0.040) was higher in the NIC group than in the placebo group. Resolution of ST segment elevation occurred in $95 \%$ and $81.67 \%$ of the patients in the NIC and placebo groups, respectively $(p=0.023)$; the MPI score of the two groups was $4.1 \pm 1.89$ and $7.3 \pm 2.65$, respectively $(p=0.014)$.

Conclusions: Early coronary administration of NIC can significantly reduce the damage in the myocardial microcirculation caused by $\mathrm{P}-\mathrm{PCl}$ and the myocardial infarct size in patients with AMI.
\end{abstract}

Key words: primary percutaneous coronary intervention, nicorandil, no-reflow.

\section{Introduction}

Primary percutaneous coronary intervention ( $\mathrm{P}-\mathrm{PCl}$ ) is currently the most effective method of treating acute myocardial infarction (AMI). However, intraoperative reperfusion injury is not rare, appearing as the no-reflow phenomenon, reperfusion-induced arrhythmia, or recurrence of chest pain [1]. The no-reflow phenomenon is an independent risk factor that affects the short-term prognosis in $\mathrm{PCl}$ and long-term cardiac death and events, and patients with this condition may experience increased myocardial infarct size, left ventricular enlargement, decreased cardiac function, malignant arrhythmia, or even death; thus, the clinical outcomes are poor [2, 3].

Nicorandil (NIC) is a $\mathrm{K}^{+}$-ATP channel opener with a unique dual mechanism of action. Nicorandil can play nitric acid ester-like roles to expand the coronary artery; conversely, it can open the $\mathrm{K}^{+}$-ATP channel on the vascular smooth muscles, thus increasing the outflow of $\mathrm{K}^{+}$ from cells, inhibiting the inflow of $\mathrm{Ca}^{2+}$, reducing the $\mathrm{Ca}^{2+}$ overload, and consequently reducing the incidence of arrhythmia; at the same time, it can expand the small coronary arteries and increase the coronary blood flow [4, 5]. Several studies have confirmed that the administration of verapamil $[6,7]$ or sodium nitroprusside $[8,9]$ in $\mathrm{P}-\mathrm{PCI}$ can improve the no-reflow phenomenon. Compared with calcium antagonists and sodium nitroprusside, NIC has no significant adverse reactions, such as significant blood pressure decline, heart rate decline, or atrioventricular block inducement [10, 11].

In the current guidelines, there is no recommendation regarding preventive medication against the no-re-

\section{Corresponding author:}

Chunguang Feng, Institute of Cardiovascular Disease, Xuzhou Central Hospital, 199 Jiefang South Road, 221009 Xuzhou, China, phone: +86 516 83985081, e-mail: cnchunguangfeng@163.com

Received: 22.11.2017, accepted: 30.01.2018. 
flow phenomenon, and no previous relevant research on NIC injection has been conducted yet on the Chinese mainland. Compared with position emission computed tomography (PET), ${ }^{99} \mathrm{TC}^{\mathrm{m}}$ - methoxyisobutyl isocyanide single-photon emission computed tomography (MIBI SPECT) has very high accuracy and specificity in assessment of viable myocardium; therefore, it is a very important noninvasive method of evaluating myocardial ischemia in and the prognosis of patients with coronary heart disease (CHD) [12-15].

\section{Aim}

In this study, we selected patients with acute anterior wall ST segment elevation myocardial infarction (AASTMI) as the study subjects, examined the effect of coronary infusion of NIC on myocardial microcirculation before the occurrence of the no-reflow phenomenon, and investigated its safety and whether it can reduce the occurrence of reperfusion injury and myocardial infarct size.

\section{Material and methods}

\section{General information}

A total of 120 patients, who were hospitalized at Xuzhou Central Hospital and underwent P-PCI for initial AASTMI from June 2014 to March 2016, were selected, including 86 men and 34 women aged $67.20 \pm 5.04$ years (range: $32-79$ years). The inclusion criteria were as follows: (1) chest pain of $>30$ min and unresponsiveness to nitroglycerin administration; (2) lead ST segment elevation in V1 to V4-V6 segments of $\geq 0.1 \mathrm{mV}$ (acute anterior wall or extensive anterior wall myocardial infarction); (3) normal or greater than normal troponin level on admission; (4) no cardiogenic shock; (5) occluded infarct-related blood vessels. The exclusion criteria were as follows: (1) history of myocardial infarction; (2) conditions that could affect the assessment of the ST segment in the electrocardiogram (ECG), such as left bundle branch block, ventricular autonomic heart rate, presence of ventricular pacemaker, etc.; (3) inability to achieve satisfactory cardiac ultrasound images; (4) age > 80 years. The physicians obtained written informed consent from each patient, and the study was approved by the hospital ethics committee. The Clinicaltrials.gov registration number is NCT02435797.

\section{Treatment}

All the patients were emergently administered $300 \mathrm{mg}$ of aspirin and $180 \mathrm{mg}$ of ticagrelor per meal and underwent trans-radial artery coronary angiography and interventional therapy. When the guide wire passed through the target lesion and reached the distal end of the coronary artery, the balloon was dilated. If the thrombus-induced load was obvious, one suction catheter was used (Thrombuster, Terumo Corporation, Japan) to aspirate the thrombi. When the antegrade blood flow was restored, each patient was classified on the basis of the thrombolysis in myocardial infarction (TIMI) grade. When the TIMI grade reached grades $2-3$, tirofiban (platelet membrane glycoprotein IIb/IIla receptor antagonist, $10 \mu \mathrm{g} / \mathrm{kg}$, trade name: Lunan Hengkang, Shandong Lunan Pharmaceutical Co., Ltd.) was injected via a coronary incision. The patients were then randomly divided into the nicorandil group (all the patients were administered NIC early, $n=60$ ) and the placebo group (all the patients were administered placebo, $n=60$ ) by the method of a random number table. Patients in the nicorandil group were intravenously injected with $2 \mathrm{mg}$ of NIC via the coronary incision (trade name: Ruikexi, Beijing Sihuan Kebao Pharmaceutical Co., Ltd.) and underwent repeated angiography after $5 \mathrm{~min}$; if the TIMI grade in the coronary artery was less than grade 3, 2 mg of NIC was re-injected to the distal end of the target lesion, and the total amount of NIC injected was never more than $6 \mathrm{mg}$. Patients in the placebo group were injected with saline ( $2 \mathrm{ml}$ each time) via the coronary incision and underwent repeated angiography after $5 \mathrm{~min}$; if the TIMI grade in the coronary artery was less than grade $3,2 \mathrm{ml}$ of saline was re-injected to the distal end of the target lesion, and the total amount of saline injected was never more than $6 \mathrm{ml}$. After related medication administration, thrombus suctioning was continued when the thrombus-induced load was still obvious; when the patients in the two groups had TIMI grades 0-2 after stent implantation, the suction catheter was re-used, or 100-200 $\mu \mathrm{g}$ of sodium nitroprusside was injected to the distal end of the target lesion. The TIMI grade and TIMI myocardial perfusion grade (TMPG) were determined at the designated time points (immediately after stent implantation (T1) and at the end of surgery (T2)). No reflow after reperfusion was defined as follows [16]: coronary angiography revealing TIMI grades 0-2 (antegrade blood flow) after $\mathrm{PCl}$, without residual stenosis, vascular wall intercalation, thromboembolism, spasm, or other mechanical obstruction. The criteria used for coronary angiography TIMI grade [17] and TMPG were in accordance with the conventional criteria [18]. The flow diagram is as follows (Figure 1).

Each patient preferred the optimized medication if no contraindication occurred, including antiplatelet, anticoagulant, statin, angiotensin-converting enzyme inhibitor, angiotensin II receptor antagonist, $\beta$-blocker, nitrate, or blood pressure and blood glucose control medications.

\section{ECG}

ECG was performed in each patient immediately on admission and $1 \mathrm{~h}$ after $\mathrm{PCl}$; thereafter, the sum of the elevated ST segment $(\Sigma S T)$ and the decline amplitude was calculated. The calculation formula was as follows: [ $\Sigma \mathrm{ST}$ (on admission) - $\Sigma \mathrm{ST}$ (after PCI)]/ $\Sigma \mathrm{ST}$ (on admission). The patients were divided into two groups: rapid decline group (decline $\geq 50 \%$ ) and ST segment continuous eleva- 
Acute anterior wall ST elevation MI patients $(n=120)$

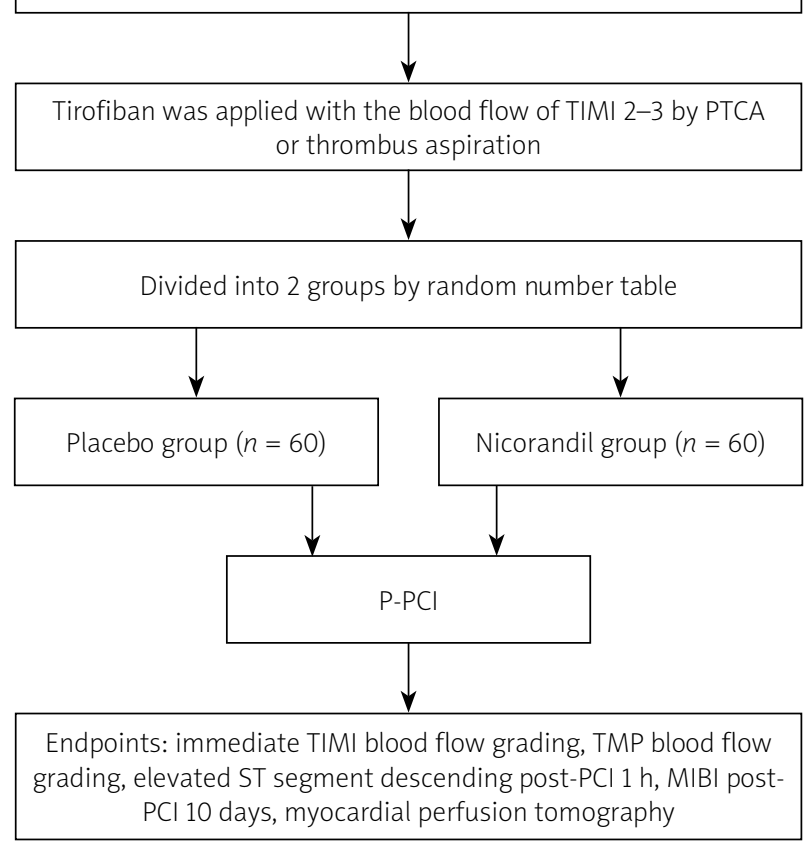

Figure 1. Flow diagram of this trial

tion group (decline $<50 \%$ ); and a decline of $<50 \%$ was considered to indicate poor reperfusion [19].

\section{Myocardial perfusion imaging (MPI)}

The two groups underwent MPI 10 days after $\mathrm{P}-\mathrm{PCI}$ using one Siemens E.cam SPECT instrument, with the imaging agent ${ }^{99} \mathrm{TC}^{\mathrm{m}}-\mathrm{MIBI}$ (radiochemical purity > 95\%); $\mathrm{MIBI}$ was provided by Jiangyuan Pharmaceutical Factory, Jiangsu Provincial Institute of Atomic Medicine, and $\sim 740 \mathrm{MBq}$ was injected into each patient's elbow vein. Based on the relationship between the distribution of the coronary artery and the anatomy of the myocardium, the left ventricle was divided into the anterior wall, anterior wall base, anterior septum, posterior septum, apex, anterolateral wall, posterior wall, lower wall, and posterior wall, which were scored in accordance with the following criteria: 0 points: normal intake of ${ }^{99} \mathrm{TC}^{\mathrm{m}}-\mathrm{MIBI}$ (the radioactivity count of this area was also set as $100 \%$ ); 1 point: radioactivity count lower than the normal level by $>25 \%$; 2 points: radioactivity count lower than the normal level by $>50 \%$; and 3 points: radioactivity count lower than the normal level by $>75 \%$. The evaluation and semi-quantitative analysis of the images were conducted independently by two experienced nuclear medicine physicians using the single-blind method.

\section{Statistical analysis}

The SPSS 17.0 software was used for the analysis; the sparse or defective segments on ${ }^{99} \mathrm{Tc}^{\mathrm{m}}$-MIBI SPECT were expressed as $\bar{x} \pm \mathrm{s}$. The measurement data between groups $A$ and $B$ were compared using the $t$-test of two independent samples; the count data were compared using the $\chi^{2}$ test (when the theoretical value was $<5$, the corrected value was used), with $p<0.05$ considered as significant.

\section{Results}

\section{Baseline data}

There was no significant difference in age, sex, risk factors of CHD (hypertension, diabetes, dyslipidemia, or smoking history), interval from onset to surgery, stent, or medication conditions between the two groups (Table I).

\section{TIMI grade and TMPG}

The grades in the nicorandil group at post-stent and post-procedure were significantly better than those in the placebo group $\left(\chi^{2}=4.62, p=0.032\right.$ and $\chi^{2}=3.93$, $p=0.047)$, suggesting that early intraoperative application of NIC can significantly reduce the occurrence of postoperative no/slow blood flow (Figure 2).

\section{Comparison of TMPG}

The number of patients with TMPG 3 in the nicorandil group at post-stent and post-procedure was significantly higher than that in the placebo group $\left(\chi^{2}=5.07, p=0.024\right.$ and $\chi^{2}=4.23, p=0.040$ ), indicating that the early intraoperative application of NIC can significantly improve the postoperative coronary microcirculation (Figure 2).

After surgery, the comparison of the ST segment decline between the two groups showed that the nicorandil group had significantly better results than the placebo group. ECG revealed a rapid ST-segment decline in the nicorandil group ( $95 \%$ vs. $81.67 \%, p=0.02$ ), indicating that the early intraoperative application of NIC can significantly improve this sensitive and specific noninvasive index, which can reflect the level of myocardial microcirculation (Table I).

The changes in the MPI score indicated that the early administration of NIC can reduce the MPI score and myocardial infarct size (Table I).

\section{Comparison of side effects}

Ten patients in the nicorandil group exhibited a transient blood pressure decline of $<10 \mathrm{~mm} \mathrm{Hg}$ after medication administration but self-recovered thereafter.

\section{Discussion}

This study found that early P-PCl-intervened administration of NIC in patients with AASTMI can significantly reduce the incidence of reperfusion injury and the myocardial infarct size without obvious side effects.

The most effective method of treating AMI is to open the infarct-related blood vessels as soon as possible to save the dying myocardium at present [20]. Early complete reperfusion against the infarct-related coronary ar- 
Table I. Comparison of baseline patient characteristics and outcomes between the two groups

\begin{tabular}{|c|c|c|c|}
\hline Parameter & Nicorandil $(n=60)$ & Placebo $(n=60)$ & $P$-value \\
\hline Age [years] & $67.53 \pm 5.11$ & $66.86 \pm 4.97$ & 0.785 \\
\hline Male & 45 & 41 & 0.418 \\
\hline Hypertension & 28 & 31 & 0.584 \\
\hline Dyslipidemia & 18 & 23 & 0.336 \\
\hline Type 2 diabetes mellitus & 25 & 18 & 0.182 \\
\hline Smoking (within 1 year) & 27 & 21 & 0.264 \\
\hline Interval from onset to $\mathrm{PCI}[\mathrm{h}]$ & $4.21 \pm 2.67$ & $4.33 \pm 2.12$ & 0.698 \\
\hline 3 of suction catheter & 5 & 12 & 0.067 \\
\hline Stent length [mm] & $22.88 \pm 7.82$ & $23.25 \pm 8.17$ & 0.726 \\
\hline Number of stents & 71 & 79 & 0.812 \\
\hline \multicolumn{4}{|l|}{ Drug: } \\
\hline Aspirin & 60 & 60 & \\
\hline Ticagrelor & 60 & 60 & \\
\hline Tirofiban & 60 & 60 & \\
\hline Nitrates & 47 & 50 & 0.487 \\
\hline Statins & 58 & 60 & 0.154 \\
\hline ACEI & 52 & 50 & 0.609 \\
\hline ARB & 8 & 10 & 0.609 \\
\hline B-receptor blockers & 53 & 54 & 0.769 \\
\hline Rapid ST-segment decline & $57(95 \%)$ & $49(81.67 \%)$ & 0.023 \\
\hline MPI score & $4.1 \pm 1.89$ & $7.3 \pm 2.65$ & 0.014 \\
\hline
\end{tabular}

Dyslipidemia: $L-L D L>70 \mathrm{mg} / \mathrm{dl}$. The drug was used during the whole duration of therapy, including admission, in-hospital stay and after discharge.

tery can reduce the myocardial infarct size, cardiac mortality, and incidence of other adverse cardiac events [21]. Owing to various reasons, TIMI grades $0-2$ would still be observed even if stenosis or occlusion had been released; although the TIMI grade reaches grade 3 in some cases, the myocardial tissue still may not achieve effective reperfusion, namely, the no-reflow phenomenon. The presence of the no-reflow phenomenon greatly diminishes the clinical benefits of $\mathrm{P}-\mathrm{PCl}$; additionally, it is a sign of severe myocardial and microvascular injuries, as well as an important factor for continuous ischemia, infarct extension, ventricular remodeling, and cardiac functional recovery disorder [1-3].

Studies have shown that patients with and without rapid normalization of the ST segment within $1 \mathrm{~h}$ of $\mathrm{P}-\mathrm{PCl}$ (elevated ST segment decline of $\geq 50 \%$ ) may exhibit significantly different prognoses. The declining amplitude of the elevated ST segment after $\mathrm{P}-\mathrm{PCl}$ can reflect the level of microcirculatory reperfusion in the related infarcted myocardium, and the more obvious the declining amplitude is, the better is the patient's prognosis [22]. ${ }^{99} \mathrm{TC}^{\mathrm{m}}-\mathrm{MIBI}$ SPECT provides direct manifestations of myocardial activity and is an important noninvasive method of assessing myocardial ischemia in and the prognosis of patients with CHD. In this study, we did not use the gold standard to assess myocardial viability, namely PET. Many studies have suggested that ${ }^{99} \mathrm{Tc}^{\mathrm{m}}-\mathrm{MIBI}$ SPECT has

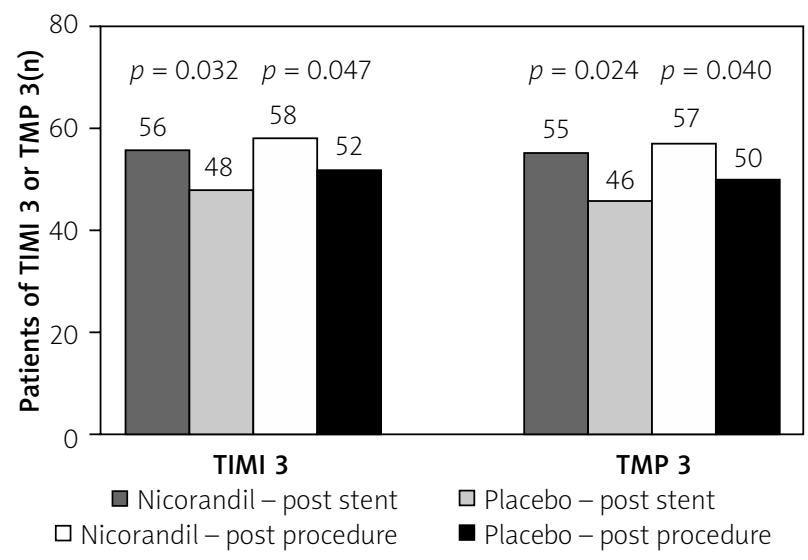

Figure 2. Comparison of target blood vessel TIMI grade 3 blood flow between the two groups. Significant improvement of target blood vessel TIMI grade 3 blood flow occurred in nicorandil group compared with placebo group 
a good correlation with PET when used to measure the local myocardial metabolic status; thus, it may have considerable clinical application value [12-15].

Previous studies have confirmed that the administration of verapamil [6, 7], adenosine [23], or sodium nitroprusside $[8,9]$ can improve the no-reflow phenomenon in percutaneous transluminal coronary angioplasty; however, these medications all yield adverse reactions, such as blood pressure and heart rate decline and atrioventricular block inducement. Moreover, the doses of such medications are limited to a certain extent. Nicorandil is a $\mathrm{K}^{+}$-ATP channel opener with a unique dual mechanism of action. Nicorandil can also play nitric acid ester-like roles to expand the coronary artery and to reduce the pre- and post-loads. Conversely, the increased outflow of $\mathrm{K}^{+}$can cause hyperpolarization of the cell membrane, shorten the duration of action potentials, inhibit the inflow of $\mathrm{Ca}^{2+}$, and reduce $\mathrm{Ca}^{2+}$ overload, thus reducing the occurrence of arrhythmia, when the $\mathrm{K}^{+}$-ATP channel on the vascular smooth muscle is open; it can also relax the vascular smooth muscles, expand the small coronary arteries, and increase coronary blood flow. Nicorandil has the same expansion effect toward the coronary arteries with different diameters, which can be more obvious in the smaller coronary arteries; compared with verapamil, adenosine, and sodium nitroprusside, it has no significant adverse reactions as in the abovementioned examples [10, 11].

Although the use of NIC at the time of PCl did not show any potential benefit on fatal and non-fatal outcomes in western countries [24], to our knowledge, no previous relevant research on NIC injection has been conducted yet on the Chinese mainland; thus, it is necessary to compare NIC with placebo to clarify its efficacy. In this study, high-concentration NIC was intravenously administered before the occurrence of the no-reflow phenomenon and exhibited its effect rapidly, in which the distal blood vessels showed a state of full expansion; NIC also further reduced the possibility of microvascular congestion and microvascular edema and spasm. The TIMI grade and TMPG in the nicorandil group were significantly superior to those in the placebo group, and after slow/ no blood flow occurred, the effect of sodium nitroprusside was also significantly better in the nicorandil group than in the placebo group; the ST segment decline and the ${ }^{99} \mathrm{TC}^{\mathrm{m}}$-MIBI SPECT score were both significantly better in the nicorandil group than in the placebo group, without significant side effects, which is consistent with the reports of other researchers [25-27]. Although administration of drugs targeting mitochondrial function, such as NIC, in STEMI patients undergoing primary $\mathrm{PCI}$ appear to have no effect on mortality, they may reduce hospital readmission for HF [28].

Campo et al. [29] found that C subunit values were higher in AMI patients with poor values of TMPG and fully or partially absent of ST-segment resolution, and $C$ subunit values were higher; left ventricle ejection fraction, wall motion score index and cumulative incidence of death and heart failure were worse in patients with elevated $C$ subunit. It can be concluded that the early application of nicorandil would reduce $C$ subunit values, so $C$ subunit can be used as a new index in future studies.

The limitations of this study were as follows: (1) This study was conducted in a single center and had a relatively small sample size, which make the results less reliable. We will conduct multi-center, large-sample, randomized controlled studies for further confirmation. (2) If SPECT and PET had both been applied to evaluate the viable myocardium, the results would have been more reliable. (3) This study compared NIC with placebo, and a pairwise comparison with active medications can further elucidate its effectiveness.

\section{Acknowledgments}

This work was funded by Science and Technology Planning Project of Xuzhou (KC14SH069).

\section{Conflict of interest}

The authors declare no conflict of interest.

\section{References}

1. Kloner RA. Does reperfusion injury exist in humans? J Am Coll Cardiol 1993; 21: 537-45.

2. Resnic FS, Wainstein M, Lee MK, et al. No-reflow is an independent predictor of death and myocardial infarction after percutaneous coronary intervention. Am Heart J 2003; 145: 42-6.

3. Morishima I, Sone T, Okumura K, et al. Angiographic no-reflow phenomenon as a predictor of adverse long-term outcome in patients treated with percutaneous transluminal coronary angioplasty for first acute myocardial infarction. J Am Coll Cardiol 2000; 36: 1202-9.

4. Taira N. Nicorandil as a hybrid between nitrates and potassium channel activators. Am J Cardiol 1989; 63: 18J-24J.

5. Treese N, Erbel R, Meyer J. Acute hemodynamic effects of nicorandil in coronary artery disease. J Cardiovasc Pharmacol 1992; 20 Suppl 3: S52-6.

6. Abu Arab T, Rafik R, El Etriby A. Efficacy and safety of local intracoronary drug delivery in treatment of no-reflow phenomenon: a pilot study. J Interv Cardiol 2016; 29: 496-504.

7. Wang L, Cheng Z, Gu Y, et al. Short-term effects of verapamil and diltiazem in the treatment of no reflow phenomenon: a meta-analysis of randomized controlled trials. Biomed Res Int 2015; 2015: 382086.

8. Yang L, Mu L, Sun L, et al. Effect of intracoronary nitroprusside injection on flow recovery during primary $\mathrm{PCl}$ in acute STEMI patients. Minerva Cardioangiol 2017; 65: 111-8.

9. Rezkalla SH, Stankowski RV, Hanna J, et al. Management of no-reflow phenomenon in the catheterization laboratory. JACC Cardiovasc Interv 2017; 10: 215-23.

10. Study S, Schmid JP, Schroeder V, et al. Comparison of the antiischaemic and antianginal effect of nicorandil and amlodipine in patients with symptomatic stable angina pectoris: the SWAN study. J Clin Basic Cardiol 1999; 14: 213-7. 
11. Kobatake R, Sato T, Fujiwara Y, et al. Comparison of the effects of nitroprusside versus nicorandil on the slow/no-reflow phenomenonduring coronary interventions for acute myocardial infarction. Heart Vessels 2011; 26: 379-84.

12. Maruyama A, Hasegawa S, Paul AK, et al. Myocardial viability assessment with gated SPECT Tc-99m tetrofosmin \% wall thickening: comparison with F-18 FDG-PET. Ann Nucl Med 2002; 16: 25-32.

13. Al Moudi M, Sun ZH. Diagnostic value of (18)F-FDG PET in the assessment of myocardial viability in coronary artery disease: a comparative study with (99m)TC SPECT and echocardiography. J Geriatr Cardiol 2014; 11: 229-36.

14. Tsai JP, Yun $\mathrm{CH}, \mathrm{Wu} \mathrm{TH}$, et al. A meta-analysis comparing SPECT with PET for the assessment of myocardial viability in patients with coronary artery disease. Nucl Med Commun 2014; 35: 947-54.

15. Rubeaux M, Xu Y, Germano G, et al. Normal databases for the relative quantification of myocardial perfusion. Curr Cardiovasc Imaging Rep 2016; 9: 22.

16. Reffelmann T, Kloner RA. The no-reflow phenomenon: basic science and clinical correlates. Heart 2002; 87: 162-8.

17. Gibson CM, Cannon CP, Piana RN, et al. Relationship of coronary flow to myocardial infarction size: two sample methods to subclassify TIMI flow grades. Circulation 1992; 86: 1-453.

18. Gibson CM, Cannon CP, Murphy SA, et al. Relationship of TIMI myocardial perfusion grade to mortality after administraion of thrombolytic drugs. Circulation 2000; 101: 125-30.

19. Tomaszuk-Kazberuk A, Musiał WJ, Dobrzycki S, et al. Normalisation of elevated ST segment predicts return of left ventricular systolic function and improved outcome in patients with acute myocardial infarction, treated with primary coronary angioplasty. Kardiol Pol 2004; 60: 541-9.

20. Kolh P, Windecker S, Alfonso F, et al. 2014 ESC/EACTS guidelines on myocardial revascularization: the Task Force on Myocardial Revascularization of the European Society of Cardiology (ESC) and the European Association for Cardio-Thoracic Surgery (EACTS). Developed with the special contribution of the European Association of Percutaneous Cardiovascular Interventions (EAPCI). Eur J Cardiothorac Surg 2014; 46: 517-92.

21. Global Use of Strategies to Open Occluded Coronary Arteries in Acute Coronary Syndromes (GUSTO IIb) Angioplasty Substudy Investigators. A clinical trial comparing primary coronary angioplasty with tissue plasminogen activator for acute myocardial infarction. N Engl J Med 1997; 336: 1621-8.

22. Yu J, Oh PC, Kim M, et al. Improved early risk stratification of patients with ST-segment elevation myocardial infarction undergoing primary percutaneous coronary intervention using a combination of serum soluble ST2 and NT-proBNP. PLoS One 2017; 12: e0182829.

23. Polimeni A, De Rosa S, Sabatino J, et al. Impact of intracoronary adenosine administration during primary $\mathrm{PCl}$ : a meta-analysis. Int J Cardiol 2016; 203: 1032-41.

24. Campo G, Pavasini R, Morciano G, et al. Data on administration of cyclosporine, nicorandil, metoprolol on reperfusion related outcomes in ST-segment elevation myocardial infarction treated with percutaneous coronary intervention. Data Brief 2017; 14: 197-205.

25. Lee HC, An SG, Choi JH, et al. Effect of intra-coronary nicorandil administration prior to reperfusion in acute ST segment elevation myocardial infarction. Circ J 2008; 72: 1425-9.
26. Wu M, Huang Z, Xie H, et al. Nicorandil in patients with acute myocardial infarction undergoing primary percutaneous coronary intervention: a systematic review and meta-analysis. PLoS One 2013; 8: e78231.

27. Chen C, Fu X, Li W, et al. Intracoronary administration of anisodamine and nicorandil in individuals undergoing primary percutaneous coronary intervention for acute inferior myocardial infarction: a randomized factorial trial. Exp Ther Med 2015; 10 : 1059-65.

28. Campo G, Pavasini R, Morciano G, et al. Clinical benefit of drugs targeting mitochondrial function as an adjunct to reperfusion in ST-segment elevation myocardial infarction: a meta-analysis of randomized clinical trials. Int J Cardiol 2017; 244: 59-66.

29. Campo G, Morciano G, Pavasini R, et al. Fo ATP synthase C subunit serum levels in patients with ST-segment elevation myocardial infarction: preliminary findings. Int J Cardiol 2016; 221: 993-7. 